TITLE:

\title{
Initial temporal and spatial changes of the refractive index induced by focused femtosecond pulsed laser irradiation inside a glass
}

\section{$\operatorname{AUTHOR}(\mathrm{S})$ :}

Sakakura, Masaaki; Terazima, Masahide

\section{CITATION:}

Sakakura, Masaaki ... [et al]. Initial temporal and spatial changes of the refractive index induced by focused femtosecond pulsed laser irradiation inside a glass. PHYSICAL REVIEW B 2005, $71(2): 024113$.

\section{ISSUE DATE:}

2005-01

URL:

http://hdl.handle.net/2433/50508

RIGHT:

Copyright 2005 American Physical Society 
PHYSICAL REVIEW B 71, 024113 (2005)

\title{
Initial temporal and spatial changes of the refractive index induced by focused femtosecond pulsed laser irradiation inside a glass
}

\author{
Masaaki Sakakura and Masahide Terazima \\ Department of Chemistry, Graduate School of Science, Kyoto University, Kyoto 606-8502, Japan \\ (Received 11 July 2004; revised manuscript received 14 October 2004; published 26 January 2005)
}

\begin{abstract}
The temporal and spatial developments of the refractive-index change in a focal region of a femtosecondlaser pulse inside a soda-lime glass is investigated by the transient lens ( $\mathrm{TrL}$ ) method with a time resolution of subpicosecond. In the TrL signal, the oscillation with about an 800-ps period is observed until about 2000 ps. In order to explain the oscillation, the thermoelastic response of a heated material by a short pulsed laser is calculated. It is found that the TrL signal calculated based on the thermoelastic calculation reproduces the observed oscillating signal very well, even though the calculated density at the focal region does not oscillate. The essential feature of the oscillation can be explained in terms of the pressure wave generation and propagation in the outward direction from the irradiated region. Based on the pressure-wave propagation and the phase-retrieval method, the temporal evolution of the refractive-index distribution inside a glass is obtained from the probe-beam deformation (TrL image) at various delay times between the pump and probe pulses. Two phases of the refractive-index increase at the laser focal region were observed in a range of 20-100 and 500-700 ps, which may cause a permanent refractive-index increase in the laser focal region inside a glass. We discuss the effect of the laser pulse duration on the material deformation process in the laser-irradiated region. This study clearly shows the initial process of the material deformation dynamics inside a glass after femtosecond laser irradiation.
\end{abstract}

DOI: $10.1103 /$ PhysRevB.71.024113

PACS number(s): 52.38.Mf

\section{INTRODUCTION}

Recently, there have been a variety of studies and applications of fabrication techniques inside transparent materials by utilizing strong electric fields of femtosecond pulsed laser beams. ${ }^{1-14}$ When a femtosecond laser pulse is tightly focused inside a transparent material such as a glass, a structural change occurs at a small volume in the material, which must be caused by the nonlinear interaction between the material and the strong laser field. When the intensity of the laser pulse is strong enough, a void or a less dense region is created due to the explosive thermal expansion. ${ }^{2,8-11}$ Because the refractive index in such a void region should be significantly different from that of the other region, a refractiveindex distribution with high contrast can be created. This technique has been utilized to produce three-dimensional (3D) optical memories ${ }^{2,8,11}$ and 3D photonic crystals, ${ }^{9}$ and other possible applications are expected. On the other hand, when the laser field is not so strong or the laser pulse is moderately focused, a structure possessing a large refractive index is observed around the laser focal region. ${ }^{1,5-8,13,14}$ This is an interesting phenomenon, and there have recently been many reports on the applications to the fabrication of various kinds of 3D micro-optics. ${ }^{1,3-8}$ This refractive-index increase suggests that the glass structure is altered or that the density of the material increases by the laser irradiation. ${ }^{1,7,8}$ Regardless of many applications, the mechanism of the structural change by a femtosecond laser inside a glass has not been well understood so far.

Much experimental research has studied the structural change inside a glass in detail using ESR measurements, ${ }^{1,6,7}$ Raman scattering measurements, ${ }^{6,7}$ photoluminescence detection, ${ }^{12}$ and observation of the morphology change $e^{8,9}$ and temperature dependence ${ }^{6}$ of the induced refractive-index change. It has been suggested that high temperature and/or high pressure localized in the laser focal region may play an important role in creating the refractive-index change. Indeed, since the lifetime of the photoexcited electrons in a glass such as silica is short $(<1 \mathrm{ps}),{ }^{15}$ a large amount of energy should be transferred to the lattice immediately after the photoexcitation, and the temperature increase and the thermoelastic stress should be induced in a very limited volume. The relaxation of the thermoelastic stress could produce the driving force for the density increase such as a compressive acoustic wave. However, other mechanisms have also been proposed, e.g., localized melting ${ }^{7,8,10}$ and production of defects or color centers by photoexcitation. ${ }^{16}$ For understanding the mechanism of the laser-induced refractiveindex increase, it may be essential to elucidate the initial step of the material response by a real-time observation at the laser-focused region. However, there has been no study that detects ultrafast time development of the temperature, pressure, and density distributions at the laser focal region as far as we aware.

Previously, we gave a preliminary report on the temporal development of the refractive index of a glass at the laserfocused region by using the transient lens ( $\mathrm{TrL}$ ) method with a time resolution of subpicosecond. ${ }^{17}$ This TrL method is a very powerful technique to observe the laser-induced refractive-index distribution with a fast time resolution by monitoring the deformation of the probe-beam shape. ${ }^{17-19}$ Because the density change should induce the refractiveindex change, the time-resolved measurement of the refractive-index distribution will provide valuable information for understanding the thermoelastic stress relaxation as well as the initial step of the refractive-index change. Inter- 


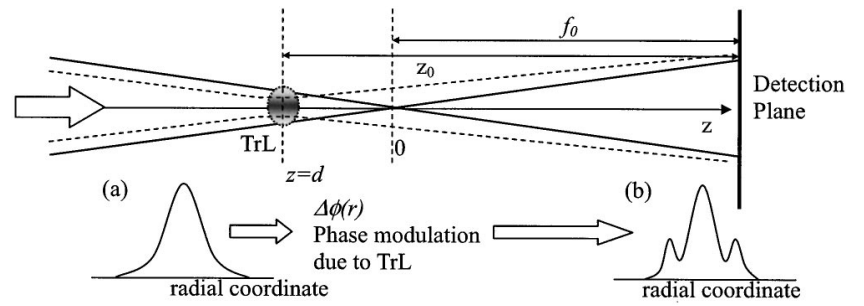

FIG. 1. Schematic illustration of the transient lens method. The beam waist of the pump pulse is located at $z=d(<0$ in this figure) and the cylindrically symmetric refractive-index distribution (transient lens; TrL) is created at this position. The probe beam propagates also along the $z$ axis from the left side and is focused at $z$ $=0$. A schematic intensity profile of the probe beam just before the TrL region is depicted in (a). After passing through $\operatorname{TrL}$, the phase of the probe light is modulated, propagates a distance of $f_{0}-d$ $\left(=z_{0}\right)$, and reaches a detection plane at $z=f_{0}$. An example of the modulated probe beam on the detection plane is shown in (b).

estingly, we discovered an oscillating TrL signal after the irradiation of a 500-fs-pulsed laser beam inside a soda-lime glass plate. ${ }^{17}$ However, the cause of the oscillation was not clear, and the refractive-index distribution and the density distribution change at the focal region were not shown.

In this study, the transient process of the refractive index around the femtosecond-laser focal region inside a glass was further investigated by the $\mathrm{TrL}$ method. The material response after the irradiation of an ultrafast laser pulse was revealed by detailed analyses of the TrL signal based on calculations of the thermoelastic response of the irradiated material in the confined region as well as a phase-retrieval analysis of the probe-beam deformation. The results clearly showed the ultrafast refractive-index changes in several phases and relaxation of the thermoelastic stress. The remainder of this paper is organized as follows. In Sec. II, the principle of the TrL method and the calculation method of the signal from a spatial nonuniform distribution of the refractive index are described. In Sec. III, we outline the experimental setup of the TrL method. In Sec. IV, we first show the oscillating TrL signal after focusing a femtosecond pulsed laser inside a glass under various experimental conditions. The signal is explained in terms of the calculated density and acoustic response of the material based on the thermoelastic equations with fast and localized heating. Next, we show the temporal evolution of the refractive-index distribution retrieved from the spatial shape of the probe beam. Finally, we discuss on the structural change shortly after the photoexcitation and the effect of the laser-pulse duration on the structural change.

\section{METHOD}

\section{A. Transient lens method}

The principle of the TrL method is similar to that of the traditional thermal lens method ${ }^{18-21}$ and schematically illustrated in Fig. 1. When a femtosecond laser pulse (a pump pulse; broken lines in Fig. 1) is focused inside a glass, the material only in the laser focal volume may absorb the laser energy through multiphoton ionization or tunneling ionization depending on the laser energy density. ${ }^{14,22}$ Following this photoexcitation, the energy of the photoexcited electrons is transferred to the lattice, resulting in temperature elevation at the photoexcited region. Due to the thermoelastic relaxation and possible chemical reactions induced by the photoexcitation, the refractive index should change, and the refractive-index distribution is created in this region. Because the induced refractive-index distribution acts as a lens, we call it a TrL. In the present $\operatorname{TrL}$ method, a probe laser beam (solid lines in Fig. 1), which is temporally delayed from the pump pulse, enters the photoexcited region coaxially. When the probe beam passes through the photoexcited region, the wavefront of the probe beam should be modulated (phase modulation) due to the created TrL. The phase modulation of the probe beam leads the modulation of the beam shape at the far field [Fig. 1(b)]. The change in the spatial pattern of the probe beam is monitored to obtain the refractive-index distribution. For example, if the $\operatorname{TrL}$ has a Gaussian shape with a negative refractive-index change, it acts as a concave lens for the probe beam. The geometric distance between the TrL region and the probe beam waist (d) is important for interpreting the deformation of the probe beam. The probe beam should be converged at the far field, when the TrL is located behind $(d<0)$ the focal point of the probe beam along the light propagation axis (Fig. 1). On the other hand, when the TrL is located before the focal point $(d>0)$, the probe beam should be expanded by the lens effect. We will explain this effect in the next section in detail.

We used two methods to measure the deformation of the spatial profile of the probe beam. The first method is similar to the traditional thermal lens method: measuring the light intensity change at the beam center at a far field by using an aperture. ${ }^{17-19}$ The transmittance change of the probe beam through an aperture placed at the beam center is generally called the TrL signal. Second, the spatial profile of the probe beam intensity is measured as an image on a detection plane. The spatial profile of the probe light intensity deformed by the TrL effect is called a "TrL image" in the present paper. Since the intensity profile of the probe beam has a circular symmetry at all delay times, we transform the TrL image into the radial distribution profile of the light intensity for the analysis.

\section{B. Calculation of TrL image and signal}

When the spatial shape of $\mathrm{TrL}$ is a Gaussian shape, the calculation method of the TrL signal is similar to that of the thermal lens method and has been already reported. ${ }^{18,19}$ However, in this study, the refractive-index distribution induced by the tightly focused laser pulse may have a complex spatial shape, because of possible complex deformations of the density due to a large temperature change. The calculation method of the TrL signal is summarized here.

We calculate the TrL image and TrL signal based on the Fresnel diffraction theory as follows. ${ }^{21,23,24}$ The geometry for the calculation is illustrated in Fig. 1. The TrL region is created by the focused pump beam at $z=d(d<0$ in Fig. 1). The probe beam propagates along the $\mathrm{z}$ axis and is focused at 
$\mathrm{z}=0$. The detection plane is located at $z=f_{0}$. When the probe beam passes through the $\mathrm{TrL}$ region, the phase of the probe beam is modulated by the refractive-index distribution. Since the spatial distributions of the laser-induced refractive-index change and the electric field of the probe beam are cylindrically symmetric around the $z$ axis, one may write the electric field of the probe beam just after the TrL region $\left[E_{1}(r)\right]$ as

$$
E_{1}(r)=E_{0}(r) \exp [j \Delta \phi(r)]
$$

where $r$ is the radial distance from the center of the beam and $E_{0}(r)$ and $\Delta \phi(r)$ are the electric field of the probe beam just before $\operatorname{TrL}$ and the phase distribution function of $\operatorname{TrL}$, respectively. According to the Fresnel diffraction theory, the propagation of the light in the homogeneous media can be calculated using the Hankel transform for the cylindrical symmetric electric field of the light. ${ }^{24}$ Therefore, after the propagation of $E_{1}(r)$ through the distance of $\mathrm{z}_{0}$, the probe electric field on the detection plane $\left[E_{S I G}(s)\right]$ is calculated by

$$
\begin{aligned}
E_{S I G}(s)= & \frac{2 \pi}{j \lambda z_{0}} \exp \left(j \pi \frac{2 z_{0}^{2}+s^{2}}{\lambda z_{0}}\right) \int_{0}^{\infty} E_{1}(r) \\
& \times \exp \left(j \pi \frac{r^{2}}{\lambda z_{0}}\right) J_{0}\left(2 \pi \frac{r s}{\lambda z_{0}}\right) r d r \\
= & \frac{2 \pi}{j \lambda z_{0}} \exp \left(j \pi \frac{2 z_{0}^{z}+s^{2}}{\lambda z_{0}}\right) \int_{0}^{\infty} E_{0}(r) \\
& \times \exp \left\{j\left(\Delta \phi(r)+\pi \frac{r^{2}}{\lambda z_{0}}\right)\right\} J_{0}\left(2 \pi \frac{r s}{\lambda z_{0}}\right) r d r,
\end{aligned}
$$

where $s$ is a radial position from the beam axis on the detection plane, $\lambda$ is the wavelength of the probe beam, $z_{0}$ corresponds to the distance between the TrL and the detection plane $\left(z_{0}=f_{0}-d\right)$, and $J_{0}(r)$ is the zeroth order of Bessel function. The intensity profile of the probe beam $I_{S I G}(s)$ on the detection plane is given by the square of $E_{S I G}(s)$,

$$
I_{S I G}(s)=\varepsilon\left|E_{S I G}(s)\right|^{2}
$$

where $\varepsilon$ is the permittivity of the air.

For the calculation of $I_{S I G}(s)$ from Eq. (2), $E_{0}(r)$ should be experimentally measured. However, measurement of both the phase and intensity in a small region inside a material is very difficult. Therefore, we estimated $E_{0}(r)$ by using the probe beam profile $\left[I_{R E F}(s)\right]$ without $\operatorname{TrL}$ as follows. When there is no TrL (no pump beam), the electric field of the probe beam at the detection plane $\left[E_{R E F}(s)\right]$ may be expressed as

$$
E_{R E F}(s)=\left\{\varepsilon^{-1} I_{R E F}(s)\right\}^{1 / 2} \exp \left[j \pi s^{2} / \lambda f_{0}\right]
$$

From the experimentally measured $E_{R E F}(s), E_{0}(r)$ can be obtained by the deconvolution of Eq. (2) with $E_{S I G}(s)$ $=E_{R E F}(s)$ and $\Delta \phi(r)=0$ :

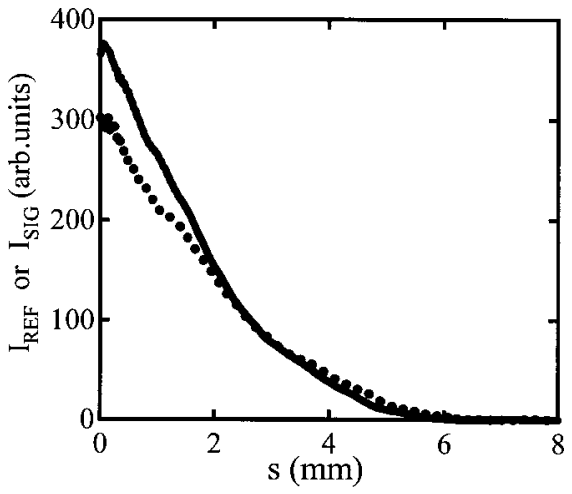

FIG. 2. An experimentally observed intensity distribution of the probe beam on the detection plane without the pump pulse $\left[I_{R E F}(s)\right.$, dotted line $]$, and typical example of the calculated light intensity on the detection plane using a concave $\operatorname{TrL}$ of $\Delta \phi(r)$ $=-4.0 \exp \left[-(r / 16 \mu \mathrm{m})^{2}\right]$ located at the position of $d=-0.06 \mathrm{~mm}$ (solid line). They are plotted against the radial position $(s)$ from the symmetric center of the probe beam.

$$
\begin{aligned}
E_{0}(r)= & \frac{1}{j \lambda z_{0}} \exp \left(-j \pi \frac{2 z_{0}^{2}+r^{2}}{\lambda z_{0}}\right) \int_{0}^{\infty} E_{R E F}(s) \\
& \times \exp \left(-j \pi \frac{s^{2}}{\lambda z_{0}}\right) J_{0}\left(2 \pi \frac{r s}{\lambda z_{0}}\right) s d s .
\end{aligned}
$$

$E_{0}(r)$ is obtained by Eqs. (4) and (5) from $I_{R E F}(s)$, and the radial intensity distribution of the TrL image $\left[I_{S I G}(s)\right]$ is calculated by using Eq. (3).

A typical example of $I_{R E F}(s)$ experimentally measured in this study is depicted in Fig. 2 (dotted line). The TrL image calculated by a phase distribution function,

$$
\Delta \phi(r)=-4.0 \exp \left[-(r / 16 \mu m)^{2}\right],
$$

with $d=0.06 \mathrm{~mm}, f_{0}=30 \mathrm{~mm}$, and $\lambda=388 \mathrm{~nm}$, which are typical values in this study, is plotted by a solid line in Fig. 2. In this case, the light intensity of the calculated beam (a solid line in Fig 2) at the central region is about 1.2 times larger than that of the reference probe beam. The result is consistent with the intensity change calculated by using the geometrical optics previously. ${ }^{18}$

Since the TrL signal intensity $\left(I_{T r L}\right)$ is defined by the light intensity at the central region of the probe beam (transmittance through an aperture), $I_{T r L}$ is calculated as follows:

$$
I_{T r L}=\int_{0}^{a} I_{S I G}(s) s d s / \int_{0}^{a} I_{R E F}(s) s d s,
$$

where $a$ is a radius of the aperture, which is located at the detection plane in Fig. 1. The TrL signal is usually normalized by the signal intensity before the pump pulse $(t<0)$ or, equivalently, $\int_{0}^{a} I_{R E F}(s) s d s .{ }^{17,18}$

\section{Calculation of refractive-index distribution from $\operatorname{TrL}$ image}

For studying the temporal and spatial change of the refractive index, we experimentally measure $I_{S I G}(s)$ (TrL image) to determine $\Delta \phi(r)$. This is an inverse problem of Eq. 


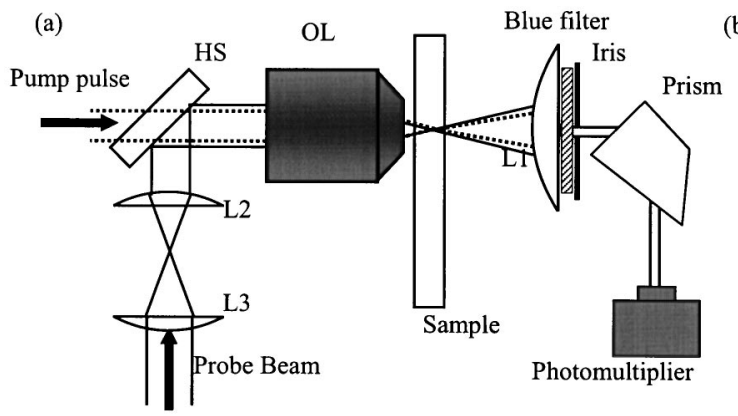

(b)

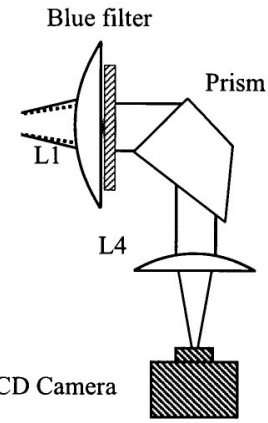

FIG. 3. Experimental setup for the TrL method (a) for detecting the TrL signal by monitoring the light intensity at the center of the beam (TrL signal), and (b) for monitoring an image of the probe beam (TrL image). HS, harmonic separator; L1, a lens with $f=30 \mathrm{~mm}$; L2, L3, and L4, lenses with $f=150 \mathrm{~mm}$; OL, a $20 \times$ microscope objective lens. The difference between (a) and (b) is the configuration after the collimation lens (L1).
(2). Since there is no phase information in $I_{S I G}(s)$, we have to fit the observed $\operatorname{TrL}$ image to determine an appropriate phase distribution function $\Delta \phi(r)$. Here, we search an appropriate $\Delta \phi(r)$ by an iterative calculation starting with an initial estimate function of $\Delta \phi(r)\left[\Delta \phi_{0}(r)\right]$ to reduce the difference between the observed image and the calculated one. At the $k$ th iteration, $I_{S I G}^{(k)}(s)$ is given by Eq. (2) with $\Delta \phi(r)$ $=\Delta \phi_{k}(r)$, and the squared error after the $k$ th iteration is defined as

$$
\varepsilon_{k}=\sum_{r^{\prime}} \sigma(s)\left[I_{S I G}(s)-I_{S I G}^{(k)}(s)\right]^{2},
$$

where $\sigma(s)$ is the weight factor for the fitting. We used $I_{R E F}(s)$ for $\sigma(s)$, because the central region in the TrL image is more important than the weaker surrounding region, which could be possibly deformed by the spherical aberration in the lens system. The next estimate of $\Delta \phi(r)\left[\Delta \phi_{k+1}(r)\right]$ is determined according to the steepest-descent method:25,26

$$
\Delta \phi_{k+1}(r)=\Delta \phi_{k}(r)-\frac{\varepsilon_{k}^{2}}{\sum_{r} \frac{\partial \varepsilon_{k}^{2}}{\partial \Delta \phi_{k}(r)}}\left\{\frac{\partial \varepsilon_{k}^{2}}{\partial \Delta \phi_{k}(r)}\right\} \gamma_{r e t},
$$

where $\gamma_{\text {ret }}(>0)$ is a retardation factor, which is adjusted to reduce the squared error. The iteration is continued until the squared error becomes smaller than a certain value, or $\Delta \phi_{k}(r)$ or $\varepsilon_{k}$ does not change after any further iterations.

\section{EXPERIMENT}

Figure 3 depicts the experimental setup for the TrL measurement. A near-infrared femtosecond laser pulse (CPA2001 Clark-MXR Inc; wavelength, $775 \mathrm{~nm}$; pulse width, $\sim 500 \mathrm{fs}$; repetition rate, $0.5-1 \mathrm{kHz}$ ) was split into two with a beam splitter. One of them was used as a pump beam and the other was passed through a $\mathrm{BBO}$ crystal to generate the second harmonic, which was used as a probe beam. The probe beam was temporally delayed by an optical delay line against the pump pulse. The collimated pump and probe beams were passed through a $20 \times$ microscope objective lens collinearly and focused inside a glass plate (typically about $500 \mu \mathrm{m}$ distant from the glass surface). All of the pump-laser power we used in this study was in a weak power range not to create a void in the glass. The probe beam after passing through the photoexcited region was collimated by a lens with a focal length of $30 \mathrm{~mm}\left(=f_{0}\right)$, and the center of the beam was passed through a small aperture (radius $1 \mathrm{~mm}$ ). The pump light was blocked by a blue filter and was completely isolated from the probe light by a prism. The transmittance of the probe beam through the aperture was monitored as a TrL signal by a photomultiplier (R-928: Hamamatsu). The signal was fed into a boxcar integrator (EG\&G: MODEL 4400-1), and plotted against the delay time of the probe beam. The focal position of the objective lens was varied by a combination of two lenses with a focal length of $150 \mathrm{~mm}$ (L2 and L3 in Fig. 3). As described in Sec. II A, the focal position of the probe beam against that of the pump beam $(d)$ is an important factor for the analysis, because the TrL signal intensity sensitively depends on the distance between the photoexcited region and the focal position of the probe beam. The distance was calculated based on the geometrical optics and the value was used for the calculation of the TrL signal.

As another detection method, the intensity profile of the probe beam (TrL image) at various delay times was measured by a CCD camera (Takex; TM-524NA) [Fig. 3(b)]. The focusing lens $(\mathrm{L} 4 ; f=150 \mathrm{~mm})$ in front of the CCD camera was adjusted to record the image of the probe beam at the position of the collimation lens (L1).

The sample was a commercial microscope slide glass plate $(25 \times 60 \times 1 \mathrm{~mm})$ made of a soda-lime glass $\left(\mathrm{SiO}_{2}\right.$ $73 \%, \mathrm{Al}_{2} \mathrm{O}_{3} 1 \%, \mathrm{CaO} 6 \%, \mathrm{MgO} 4 \%, \mathrm{Na}_{2} \mathrm{O} 15 \%, \mathrm{Fe}_{2} \mathrm{O}_{3}$ $>1 \%, \mathrm{SO}_{3}, \mathrm{~K}_{2} \mathrm{O} 1 \%$ ). Because the TrL signal by an irreversible reaction was measured in this experiment, the glass sample was moved by a computer-controlled stepping motor during data acquisition to avoid multi-irradiation at the same spot.

\section{RESULT AND DISCUSSION}

\section{A. Transient lens signal}

Figure 4 shows the TrL signal after the pump pulse (laser power $0.55 \mu \mathrm{J} /$ pulse; pulse width $500 \mathrm{fs}$ ) was focused inside the glass plate at $d=-0.06 \mathrm{~mm}$. The essential features of the TrL signal have been reported previously. ${ }^{17}$ We first briefly summarize the features. In the shorter time range ( -2 to 10 ps) shown in the inset of Fig. 4, a negative signal (decrease of the transmittance through the aperture of $0.5 \mathrm{~mm}$ radius) appears immediately after the irradiation. This signal decays very fast, and the light intensity returns back to that before the irradiation. The time duration of this signal is almost 


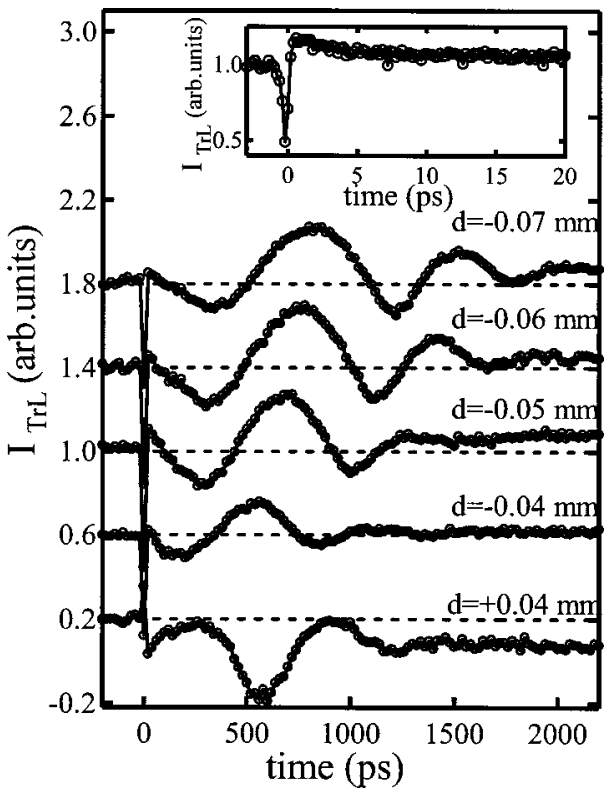

FIG. 4. Observed TrL signals after the irradiation of the femtosecond laser pulse inside a glass at various distances between $\operatorname{TrL}$ and the focal position of the probe beam $(d)$. The plots are offset vertically for clarity and the baseline $\left(I_{\mathrm{TrL}}=1.0\right)$ for each signal is depicted by a broken line. The inset figure shows the TrL signal in a short time range measured at $d=-0.06 \mathrm{~mm}$.

equal to that of the pump pulse ( $\sim 500 \mathrm{fs})$. Since $d$ is negative in this case, the decrease of the transmittance indicates the creation of convex lens at the photoirradiated region. Considering the ultrafast response of the signal, we attribute the main origin of the signal to the electronic response of the optical Kerr effect (OKE), which has been reported in the liquid phase previously. ${ }^{19}$ The sign of the refractive index change $(\delta n>0)$ agrees with this assignment. ${ }^{15,19}$

Another type of the TrL signal appears in a long time range. This signal intensity oscillates with a time period of about 800 ps. For example, at $d=-0.06 \mathrm{~mm}$, the transmittance of the probe light decreases gradually in the first $250 \mathrm{ps}$ and oscillates several times until about $2000 \mathrm{ps}$. Intuitively, if we consider only the Gaussian-type refractive-index distribution as the source of the lens signal, the oscillation suggests the oscillating refractive index at the center of the beam between $\delta n>0$ and $\delta n<0$. If this interpretation is correct, the density of the glass at the photoirradiated region may increase and decrease several times. However, we do not think this interpretation is correct, because of the following reason. Figure 4 shows the TrL signals under various $d$ with the same pump-pulse energy. This result clearly shows that the phase and the frequency of the oscillation depend on $d$. If the oscillation of the density at the photoirradiated region is the cause of the TrL signal oscillation, the phase and the frequency of the TrL signal oscillation must be determined by the material response and must be independent of the geometric distance $d$. Therefore, we exclude the possibility of the density oscillation at the focal region.

In order to find the origin of the oscillation, it is preferable to directly determine the spatial distribution of the refractive index $[\Delta \phi(r)]$ from the intensity profile of the probe beam
$\left[I_{S I G}(s)\right]$. The principle of the retrieving method is described in Sec. II C. However, we found that this retrieval calculation is very sensitive to the initial estimate function for the iterative calculation $\left[\Delta \phi_{0}(r)\right]$ and that it is almost impossible to obtain a reliable profile without knowing the origin of the oscillation. In order to resolve this dilemma, we have to guess a plausible origin of the TrL signal first.

\section{B. Calculated TrL signal based on the thermoelastic relaxation model}

Considering that the focusing of the femtosecond laser pulse inside a glass leads the photoexcitation of the material and that the thermal energy should be released by the nonradiative relaxation, we may reasonably speculate that the thermoelastic response of the material causes the TrL signal. Based on this idea, we calculated the material response after the laser-induced heating and then calculate the TrL signal.

First, the material response after the laser-induced temperature change was calculated using the thermoelastic wave equation. ${ }^{27-29}$ Under the assumption that the temperature distribution induced by the tightly focused laser pulse has spherical symmetry, the thermoelastic wave equation in an isotropic solid can be expressed by only one spatial variable, $R$, the radial position from the symmetric center of the temperature distribution:

$$
\begin{aligned}
\rho \frac{\partial^{2} u(t, R)}{\partial t^{2}}= & \frac{Y(1-\sigma)}{(1+\sigma)(1-2 \sigma)}\left(\frac{\partial^{2} u(t, R)}{\partial R^{2}}+\frac{1}{R} \frac{\partial u(t, R)}{\partial R}\right) \\
& -\frac{Y}{(1+\sigma)(1-2 \sigma)} \frac{u(t, R)}{R^{2}}-\frac{Y \beta}{3(1-2 \sigma)} \frac{\partial T(t, R)}{\partial R},
\end{aligned}
$$

where $\rho$ is the density, $u(t, R)$ stands for the displacement along the radial direction, $Y$ is Young's modulus, $\sigma$ is Poisson's ratio, $\beta$ is the thermal expansion coefficient, and $T(t, R)$ is the laser-induced temperature distribution. We solved Eq. (9) numerically by the finite element calculation technique ${ }^{30}$ with the following initial and boundary conditions:

$$
\begin{gathered}
u(t=0, R)=0, \\
u(t, R=0)=u(t, R=\infty)=0
\end{gathered}
$$

The density in the irradiated region $[\rho(t, R)]$ is given by

$$
\rho(t, R)=\rho_{0}\left(\frac{\partial u(t, R)}{\partial R}+1\right)^{-1},
$$

where $\rho_{0}$ is the density before the laser irradiation. The temperature distribution is assumed to be a Gaussian spatial shape and rises exponentially with a lifetime of $\tau_{\text {heat }}$, which is called a heating time:

$$
T(t, R)=T_{0}+\Delta T \exp \left(-\frac{R^{2}}{w_{\text {temp }}^{2}}\right)\left\{1-\exp \left(-t / \tau_{\text {heat }}\right)\right\} \quad(t>0),
$$

where $T_{0}$ is the ambient temperature, $\Delta T$ is the temperature increase, and $w_{\text {temp }}$ is the width of the temperature distribu- 

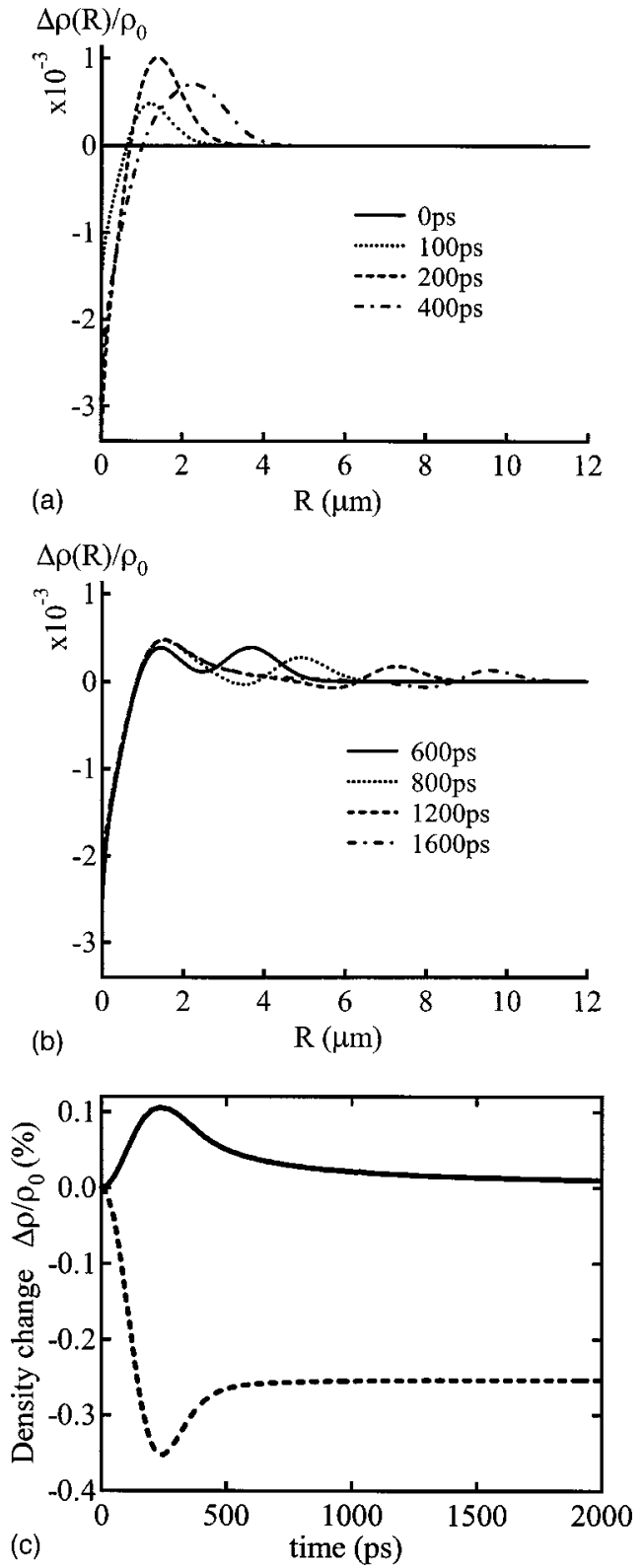

FIG. 5. (a), (b) Calculated spatial distribution of density changes at various times by Eqs. (9) $-(12)$ with $\tau_{\text {heat }}=0.5$ ps. (c) The broken and the solid lines depict the temporal evolutions of the density change at the center and the peak of the pressure wave, respectively.

tion, which may not be the same as the spatial width of the pump pulse due to the multiphoton excitation.

Using Eqs. (9)-(12), we calculated the density distribution change of the material after the photoexcitation in the confined local region. For simplicity, the equations were solved under assumptions that all mechanical constants are spatially uniform and independent of temperature. Under these assumptions, the qualitative feature of the calculated density change does not depend on the magnitude of $\Delta T$, but only the amplitude of the change does. We used the pulse width of the laser as $\tau_{\text {heat }}(=0.5 \mathrm{ps})$ throughout this paper. For example, Figs. 5(a) and 5(b) show the normalized density distributions $\left[\rho(t, R) / \rho_{0}\right]$ of the soda-lime glass $\left[\rho_{0}\right.$ $=2.51 \mathrm{~g} \mathrm{~cm}^{-3}, Y=72.9 \mathrm{GPa}, \sigma=0.21$, and $\left.\beta=72 \times 10^{-7} \mathrm{~K}^{-1}\right]$ at various times after heating with $w_{\text {temp }}=1.0 \mu \mathrm{m}$ and $\Delta T$ $=1000 \mathrm{~K}$. Initially, the density around the center gradually decreases because of the thermal expansion at the central region. Simultaneously, the density around $R=1.2-2.0 \mu \mathrm{m}$ increases until $t=240 \mathrm{ps}$. After about $400 \mathrm{ps}$, a pressure wave becomes apparent around the center and propagates outwards with the velocity of $5.8 \mu \mathrm{m} / \mathrm{ns}$. As expected, the velocity obtained here is nearly the same as that calculated from ${ }^{27}$ $v_{a c}=\sqrt{Y(1-\sigma) / \rho(1+\sigma)(1-2 \sigma)}(=5.72 \mu \mathrm{m} / \mathrm{ns})$ and close to the experimentally reported longitudinal sound velocity in a soda-lime glass $(\sim 5.84 \mu \mathrm{m} / \mathrm{ns}$ at room temperature $){ }^{31}$ The width of the pressure wave is about $0.8 \mu \mathrm{m}$ after $600 \mathrm{ps}$, which is about $80 \%$ of the radius of the temperature distribution. To clearly show the temporal evolution of the density change, the density change at the center and the peak amplitude of the pressure wave are plotted in Fig. 5(c). The density at the center (broken line) becomes minimum at $240 \mathrm{ps}$ and recovers slightly between 240 and 500 ps. In contrast, the behavior of the peak corresponding to the pressure wave (solid line) is opposite to that at the center.

We next calculate the TrL signal from the $\Delta \rho(t, R)$ obtained under various $w_{\text {temp }}\left[=\rho(t, R)-\rho_{0}\right]$. It is assumed that the phase distribution $\Delta \phi(t, r)$ is proportional to the total density change $\Delta \rho(t, R)$ integrated along the optical path:

$$
\Delta \phi(t, r)=\alpha \int \Delta \rho(t, R) d z
$$

where $\alpha$ is a constant, which is determined by the wavelength of the probe beam and the refractive index of a material.

The calculated TrL signals from Eqs. (3) and (13) and $\Delta \rho(t, R)$ at various $w_{\text {temp }}$ with the parameters of $d$ $=-0.06 \mathrm{~mm}, \lambda=388 \mathrm{~nm}$, and $f_{0}=300 \mathrm{~nm}$ are depicted in Fig. 6. For qualitative discussion, we normalize $\Delta \phi(t, r)$ at the positive maximum amplitude by adjusting $\alpha$. Interestingly, although there is no oscillating behavior in $\rho(t, R)$ [Figs. 5(a) and 5(b)], the calculated TrL signal oscillates (Fig. 6). The feature of the oscillation depends on the temperature distribution. For example, with increasing $w_{\text {temp }}$, the period of the oscillation becomes longer.

The TrL signals at various $d$ are shown in Fig. 7 with $w_{\text {temp }}=1.5 \mu \mathrm{m}$. Interestingly, we found that the calculated signals at various $d$ reproduce well the experimental observed $d$ dependence described in the previous section (Fig. 4). This agreement strongly supports that the density change calculated from the thermoelastic equation mimics the origin of the oscillating TrL signal. Since the period of the oscillation depends on $w_{\text {temp }}$ as shown in Fig. 6, $w_{\text {temp }}$ under the present experimental condition may be estimated by comparing the experimental profile to the calculated one (Fig. 6). Comparing the data of Fig. 4 with Fig. 6, we found that the calculated signal with $w_{\text {temp }}=1.0-1.5 \mu \mathrm{m}$ reproduces the essential feature of the observed TrL signal.

Qualitatively, the origin of the oscillation of the TrL signal should be the propagation of the pressure wave, because the central density dip does not depend on time so much in a longer time range $(t>600 \mathrm{ps})$ [Fig. 5(b)]. We confirm this 


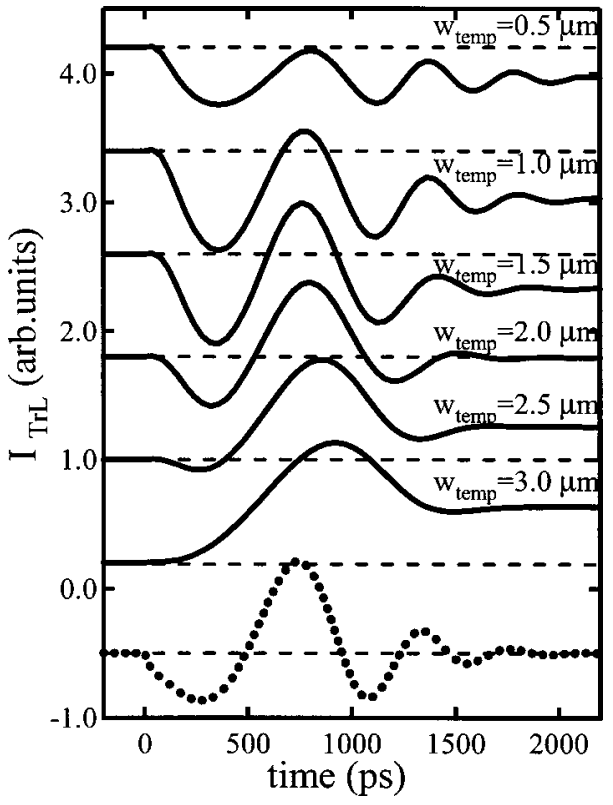

FIG. 6. Calculated TrL signals from the density distribution obtained by the thermoelastic equation with various widths of the temperature distributions $\left(w_{\text {temp }}\right)$. The dotted line is the TrL signal calculated from the function, which contains only the contribution of the acoustic wave propagation with a width of $1.0 \mu \mathrm{m}$ [Eq. (14)]. All the plots are vertically offset for clarity and the baseline $\left(I_{\mathrm{Trl}}\right.$ $=1.0$ ) for each signal is depicted by a broken line.

speculation by calculating the TrL signal from only the acoustic contribution, which may be expressed by

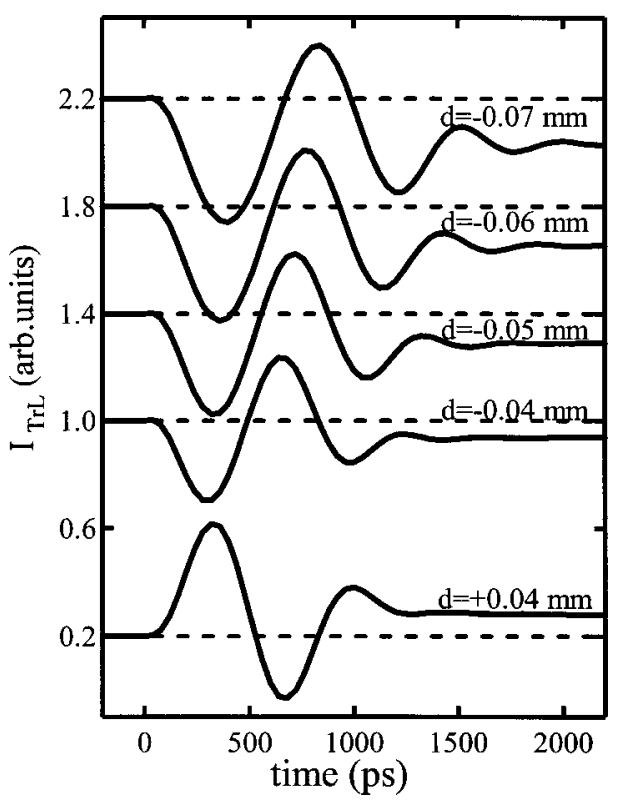

FIG. 7. Calculated TrL signals by $\Delta \rho(t, R)$ from the thermoelastic equation [Eq. (9)] with $w_{\text {temp }}=1.5 \mu \mathrm{m}$ at various distances between TrL and the focal position of the probe beam $(d)$. All the plots are vertically offset for clarity and the baseline $\left(I_{T r L}=1.0\right)$ for each signal is depicted by a broken line.
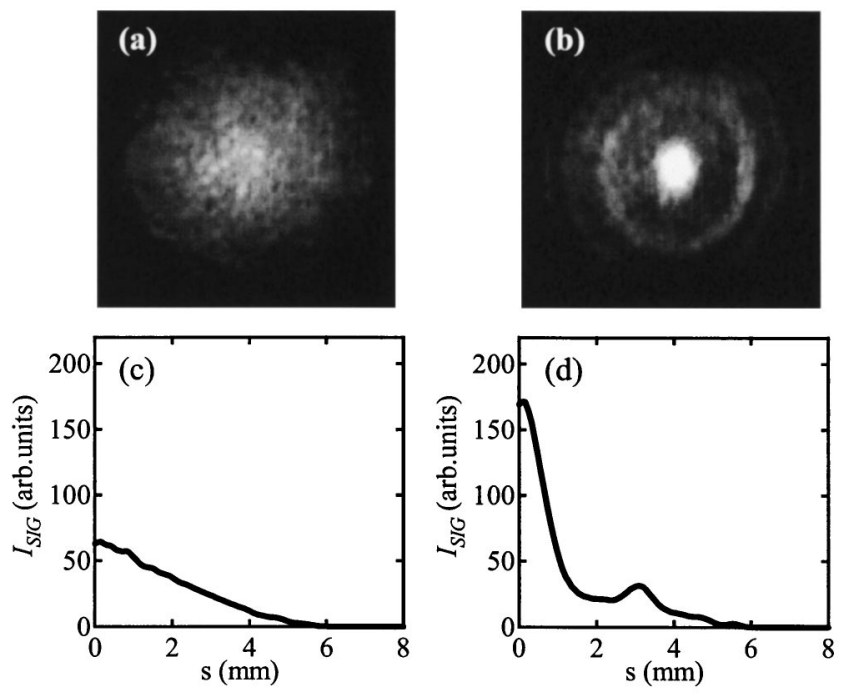

FIG. 8. Images of the probe beam detected by the CCD camera at the time delays of (a) -40 ps and (b) 720 ps. Because all the images are circularly symmetric, the intensities of the probe beam are plotted against the radial position from the symmetric center. (c) and (d) are the radial intensity distributions of the $\operatorname{TrL}$ signals at $-40 \mathrm{ps}$ and $720 \mathrm{ps}$, respectively.

$$
\Delta \phi(t, r)=\exp \left[\left\{\left(r-v_{a c} t\right) / w_{a c}\right\}^{2}\right],
$$

where $v_{a c}$ and $w_{a c}$ are the velocity and width of the pressure wave, respectively. This function mimics the phase distribution due to the pressure wave propagation. The dotted line in Fig. 6 is the simulated TrL signal using Eq. (14) with $w_{a c}$ $=1.0 \mu \mathrm{m}$ and $v_{a c}=5.8 \mu \mathrm{m} / \mathrm{ns}$. It clearly shows that the origin of the oscillation is the pressure-wave propagation.

\section{The refractive-index distribution from the TrL image}

In the preceding section, we showed that the pressurewave propagation can reproduce the essential feature of the observed TrL signal. We next try to determine $\Delta \phi(t, r)$ by the curve fitting of the TrL image at various delay times. Typical TrL images obtained under a condition of $d=-0.06 \mathrm{~mm}, f$ $=30 \mathrm{~mm}$, and $0.55 \mu \mathrm{J} /$ pulse at $-40 \mathrm{ps}$ and $720 \mathrm{ps}$ are shown in Figs. 8(a) and 8(b), respectively. The TrL image is transformed into the radial distribution profile of the light intensity as shown in Fig. 8(c) and 8(d). We fit this distribution to obtain $\Delta \phi(t, r)$.

As mentioned previously, it is important to select an appropriate initial estimate function $\Delta \phi_{0}(t, r)$ for searching reliable $\Delta \phi(t, r)$ in the phase-retrieval calculation. Since it was found that the physical origin of the oscillating TrL signal is the pressure-wave generation and propagation due to the thermoelastic relaxation of the laser-irradiated material in the preceding section, we express $\Delta \phi_{0}(t, r)$ in terms of the pressure-wave propagation. Considering the spatial and temporal profile shown in Fig. 5, we used the following function as an initial estimate function for fitting the TrL images:

$$
\Delta \phi_{0}(t, r)=-A_{1} \exp \left[-\left(r / w_{1}\right)^{2}\right]+A_{2} \exp \left[\left\{\left(r-v_{a c} t\right) / w_{a c}\right\}^{2}\right],
$$

where the first term represents the central dip due to thermal expansion and the second one is the contribution of the 
(a)

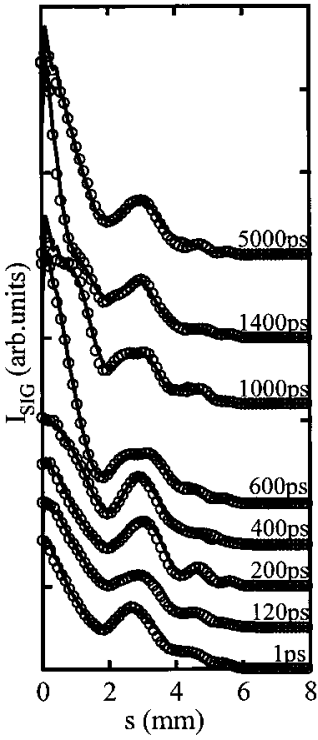

(b)

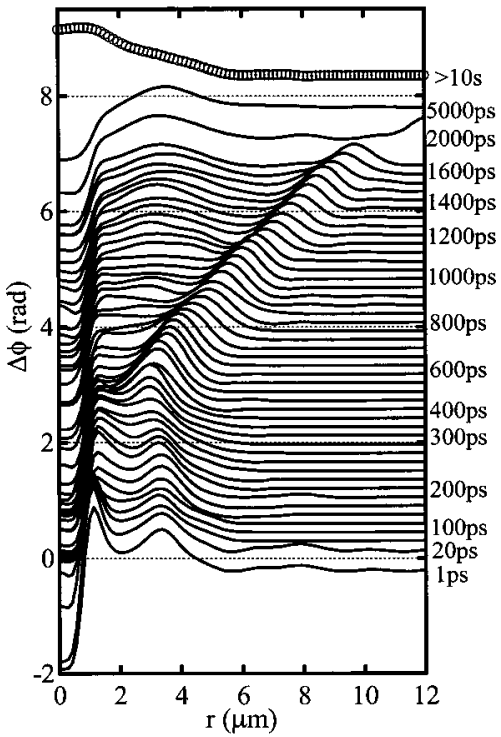

FIG. 9. (a) The spatial intensity profiles of the probe beam $\left[I_{S I G}(s)\right]$ at various delay times (open circles) and their fittings by the phase retrieval calculation (solid lines). The baseline $\left(I_{\mathrm{SIG}}=0\right)$ in each profile is on the same level as the point at $s=8 \mathrm{~mm}$. (b) The temporal evolution of the phase distribution function $\Delta \phi(t, r)$ obtained by this phase-retrieval calculation of the TrL images. All traces are offset for clarity; the baseline $(\Delta \phi=0)$ in each trace is on the same level as the point at $r=12 \mu \mathrm{m}$. The open circles depict the phase distribution obtained at more than $10 \mathrm{~s}$, after the photoirradiation of about 1000 pulses with a $1-\mathrm{kHz}$ repetition rate. The positive phase change at $t>10 \mathrm{~s}$ indicates that the refractive index increases by the present femtosecond laser irradiation.

pressure-wave propagation. An appropriate set of the parameters of $A_{2}, v_{a c}$ and $w_{a c}$ is used for reproducing the amplitude and frequency of the oscillation of the TrL signal (e.g., $A_{2}$ $=0.5, v_{a c}=5.8 \mu \mathrm{m} / \mathrm{ns}$, and $w_{a c}=1.0 \mu \mathrm{m}$ for the data of Fig. 9). The other parameters, $A_{1}$ and $w_{1}$ are determined to reproduce the TrL image at each time.

Figures 9(a) and 9(b) show the fitted radial intensity distribution of the TrL images and final $\Delta \phi(t, r)$ obtained from the fitting, respectively. The results of the phase-retrieval calculation reproduce the intensity distribution very well at all delay times. Especially, the intensity distribution in the central region is well reproduced. On the other hand, the points at larger $s$ are poorly fitted, because $I_{R E F}(s)$ with a nearly Gaussian function shape was used as the weight factor $[\sigma(s)]$ in the calculation. This choice of $\sigma(s)$ is important for reliable retrieves, because the light intensity at large $s$ is weak and easily disturbed by the aberration. Although each datum in Fig. 9(a) at various times was fitted independently, the phase distribution function $\Delta \phi(t, r)$ shown in Fig. 9(b) changes continuously with time. No abrupt change in $\Delta \phi(t, r)$ supports the reliability of the fitting. We also showed the phase distribution of the photoexcited region after $10 \mathrm{~s}$ of the laser irradiation. Because the lens effect by a final structure with only one pulse was too slight to obtain the phase distribution, we showed the phase distribution after about 1000 shots of fs-laser pulses with $1 \mathrm{kHz}$. The phase

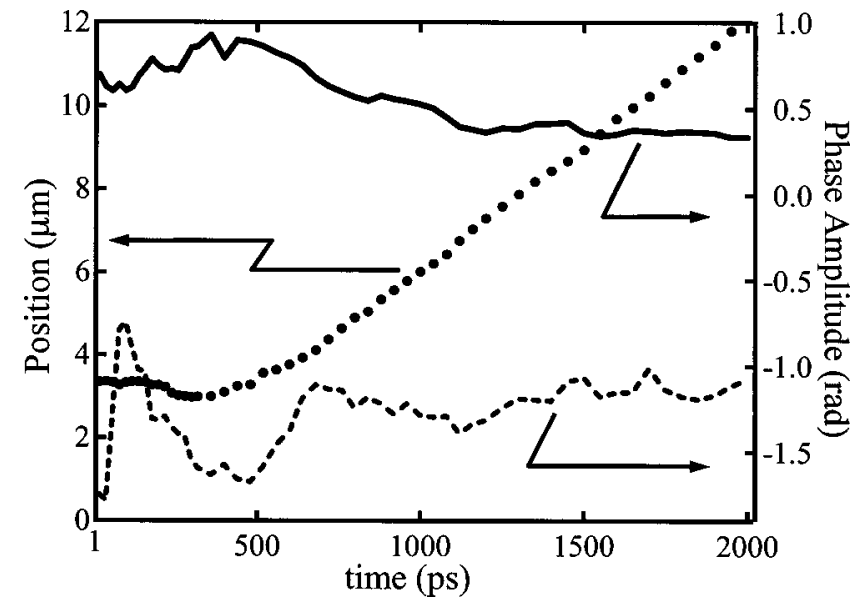

FIG. 10. The temporal profiles of the amplitude of the phase change due to the pressure wave (solid line) and that at the center of the laser-irradiated region (broken line). The dotted line depicts the position of the pressure wave.

distribution has a positive shape, which indicates that the refractive index increased after fs-laser irradiation.

The change of $\Delta \phi(t, r)$ immediately after the photoexcitation is dramatic: the phase distribution at 1 ps consists of a large hollow with a diameter of about $0.9 \mu \mathrm{m}$ and sharp and broad positive peaks at 1.2 and $3.5 \mu \mathrm{m}$, respectively. To clearly show the temporal evolution of $\Delta \phi(t, r)$, the phase at the center and that at the peak of the pressure wave are plotted against the delay times in Fig. 10. The phase at the center (broken line), which is negative immediately after the photoexcitation, increases from 20 to 100 ps, and gradually decreases until 480 ps. After 500 ps, the central phase increases until $700 \mathrm{ps}$ and remains nearly constant in a longer time range (700-2000 ps). On the other hand, the phase at the position of the pressure wave (solid line) gradually increases until 480 ps and decays slowly.

It is particularly interesting to note the initial rapid increase of the phase at the center (20-100 ps). No such change is predicted in the thermoelastic calculation and the origin is not clear at present. However, we may speculate the origin as the structural change of the glass at a high temperature under a high pressure, because of the following reasons. Since it is reported that the lifetime of plasma in $\mathrm{SiO}_{2}$ is shorter than $1 \mathrm{ps}$, and the energy of photoexcited electrons is transferred to the lattice within 1 ps in $\mathrm{SiO}_{2},{ }^{15}$ the temperature of this region is certainly elevated very rapidly and dramatically. However, the density change cannot occur until the pressure wave propagates away from the irradiated region, which is in an order of 400 ps. Hence, the hightemperature region is confined in a limited volume, and it suggests that the pressure in this region at this time should be very high as estimated in the next section. Under such conditions, the rearrangement of the atoms in the glass could take place, and the observed change in $\Delta \phi(t, r)$ in the fast time region (20-100 ps) may reflect this structural change at high temperature and under high pressure. ${ }^{32}$

Between 300 and 500 ps, the phase around $3.0 \mu \mathrm{m}$ increases with gradually changing its position outward (dotted line in Fig. 10). This increase corresponds to the pressure- 
wave generation. After $500 \mathrm{ps}$, the positive peak due to the pressure wave propagates outward with a constant velocity $(5.8 \mu \mathrm{m} / \mathrm{ns})$. The good agreement of this velocity with the longitudinal sound velocity in the soda-lime glass at room temperature $(5.8 \mu \mathrm{m} / \mathrm{ns})$ suggests that the temperature of this region, in which the pressure wave propagates with the constant velocity, is not elevated by the laser irradiation and the pressure wave behaves as an elastic wave. ${ }^{31}$ The width of the pressure wave was $0.98 \mu \mathrm{m}$ and the phase shift due to the pressure wave was 0.5 on average.

Another interesting point in Fig. 10 is that the phase at the center increases from 500 to $700 \mathrm{ps}$, and this increase occurs simultaneously with the onset of the pressure-wave propagation. From the thermoelastic calculation (Fig. 5), this phase increase should be attributed to the density increase. This density increase is caused by the generation and propagation of the pressure wave. This gives a real-time observation of the density increase at the fs-laser-irradiated region inside a glass induced by the pressure wave. This density increase will be discussed in a later section (IV E).

Although the qualitative features of the phase distribution (Fig. 10) look similar to those of the density change calculated by the thermoelastic equation [Fig. 5(c)], the response time is different. In the thermoelastic calculation, the central density becomes minimum at 290 ps. However, experimentally, this time is $480 \mathrm{ps}$, which is about twice larger than the expected value. This difference may be due to the assumption we used in the thermoelastic calculation; mechanical constants (Young's modulus, Poisson's ratio, and so on) do not depend on temperature. The slower response of the density in the experiment can be explained by the temperature dependence of Young modulus. It is well known that Young modulus of the soda-lime glass decreases with increasing the temperature. ${ }^{33}$ Since the sound velocity in solid is proportional to the square root of the Young modulus, the mechanical response is expected to be slower in a solid with smaller Young modulus. Therefore, the slower time response of the density change in the experiment indicates the smaller Young modulus in the laser-irradiated region, because of the temperature rise.

After the pressure wave escapes away from the probe beam region $(t>2000 \mathrm{ps})$, there is little temporal change in $\Delta \phi(t, r)$ as evident by the comparison of the phase distributions between at 2000 and 5000 ps in Fig. 9(b). The small temporal change in $\Delta \phi(t, r)$ after 2000 ps might suggest the stable structure in the photoexcited region at 2000 ps. However, the phase distribution after $10 \mathrm{~s}$ (the open circles in Fig. 9), which should reflect the completely relaxed structure, shows a positive refractive-index change, while that at 5000 ps shows a negative change. It indicates that the photoexcited material is not completely relaxed at $5000 \mathrm{ps}$. The difference is easily explained by the localized thermal energy in the irradiated region at $5000 \mathrm{ps},{ }^{17}$ because the thermal diffusion time is calculated to be much longer $(>1 \mu \mathrm{s})$ than 5000 ps. ${ }^{8,27,28}$ Therefore, the little difference in $\Delta \phi(t, r)$ during 2000-5000 ps suggests that the laser-irradiated region reaches mechanical equilibrium under the quasistatic nonuniform temperature distribution by 2000 ps.

\section{Estimates of density, pressure, and temperature changes}

In this section, magnitudes of the density, pressure, and temperature by laser irradiation is estimated from the experimentally obtained phase change. From Fig. 10, the phase shift due to the pressure wave was calculated to be $\Delta \phi$ $=0.5$ on average. Under the assumption that the refractive index is proportional to the density change, the phase shift can be written as ${ }^{23}$

$$
\Delta \phi=2 \pi \frac{\Delta n l}{\lambda}=2 \pi\left(n_{0}-1\right) \frac{\Delta \rho}{\rho_{0}} \frac{l}{\lambda},
$$

where $\Delta n$ is refractive-index change, $n_{0}$ and $\rho_{0}$ are the refractive index and density before photoirradiation, and $l$ is the length of the pressure wave along the probe beam axis. Since other previous studies showed that the length of the structural change along the pump beam is less than $20 \mu \mathrm{m},{ }^{5,9}$ we assumed $l=20 \mu \mathrm{m}$. Using the experimental values for $n_{0}$ and $\lambda, \Delta \phi=0.5$ corresponds to the density change $\left(\Delta \rho / \rho_{0}\right)$ of at least $0.2 \%$.

The pressure $(p)$ due to the density change $\left(\Delta \rho / \rho_{0}\right)$ can be calculated by ${ }^{27,28}$

$$
p=\frac{Y}{3(1-2 \sigma)}\left(\frac{\Delta \rho}{\rho_{0}}\right) .
$$

Using this equation, we estimate the pressure due to the density change is about $83 \mathrm{MPa}$. Since the velocity of the pressure wave is nearly same as the longitudinal sound velocity, the pressure should not be large enough to affect the structure in nonirradiated region.

In order to reproduce the $0.2 \%$ change in the acoustic density by the thermoelastic calculation, the temperature at the center of the laser beam should be larger than at least $4000 \mathrm{~K}$, even if we assume that the material response is still in the linear region. The elevation of the temperature to 4000 $\mathrm{K}$ induces the pressure increase of $0.3 \mathrm{GPa}$ at the beam center from an equation of

$$
p=\frac{Y}{3(1-2 \sigma)} \beta \Delta T .
$$

A molecular dynamics (MD) simulation study of fused silica under high-pressure ${ }^{34}$ and shock experiments ${ }^{31}$ showed that the structural transformation (for example, elastic to plastic and change in the number of Si rings) occurs above $8 \mathrm{GPa}$ at $300 \mathrm{~K}$. Even though this transformation pressure is larger than that calculated from this experiment $(0.3 \mathrm{GPa})$, it is plausible that the high temperature at the laser focal region may induce the conformational change in the glass under this pressure.

\section{E. Material deformation process and effect of the pulse duration}

We observed the structure with high refractive index in the fs-laser focal region after irradiation (opened circles in Fig. 9). Previously, the origins of the refractive-index increase were studied and attributed to densification of the irradiated material, color center creation, or defect formation. ${ }^{1,6-8}$ The color center could account for the 
refractive-index increase as expected from the KramersKronig relation between light absorption and refractive index. ${ }^{16,18}$ In fact, a recent MD simulation by Sen and Dickinson ${ }^{16}$ revealed that the color center is created after photoexcitation of a silica glass and refractive-index increase. However, Streltsov et al. ${ }^{6}$ showed that only the induced color center cannot account for the observed refractive-index increase based on annealing experiments at several temperatures. The micro-Raman observation by Chan et $a l .{ }^{7}$ confirmed that the densified structure is created in the laser focal region. Therefore, it may be appropriate to consider that the macroscopic structural change (i.e., densification) should be important for the refractive-index change. The observation of the material deformation process in this study should provide a clue to elucidate the mechanism of the structural change.

It is now widely recognized that the effect of the laser irradiation of femtosecond pulses inside a glass is completely different from that of nanosecond laser pulses. In particular, the small and smooth structure with a high refractive index after the laser light irradiation has been observed only using femtosecond pulsed laser. How does the pulse duration of the laser affect the structural change in the laser focal region? Several researchers have suggested that temperature in the fs-laser focal region becomes as high as the glass transition temperature (for example, the estimated temperature after fslaser irradiation is as high as $1800 \mathrm{~K}$ for a fused silica ${ }^{6-8}$ ) based on observations of Raman scattering, ${ }^{7}$ photoluminescence, ${ }^{12}$ and morphology of the final structure. ${ }^{8}$ It has been sometimes considered that a laser pulse with several picoseconds duration may be required for creating a localized high-temperature region, which may be the origin of the densification. ${ }^{7,8}$ However, a picosecond pulse is not actually necessary for the creation of the localized high temperature (temperature confinement), because the thermal diffusion time from a $1-\mu \mathrm{m}$ region is much longer than $1 \mathrm{~ns}$ as shown in Sec. IV C. Therefore, a temperature elevation in a localized region can be achieved even by a nanosecond laser pulse.

On the other hand, the thermoelastic relaxation accompanied with a pressure wave observed in this study should be a unique phenomenon with a femtosecond laser pulse. Ultrashort laser irradiation of the material induces temperature elevation in the irradiated region with no displacement of the material. The nonequilibrium due to the sudden temperature change with no displacement causes large mechanical stress (thermoelastic stress confinement) in the irradiated region, and the following stress relaxation generates stress wave. Some studies on laser ablation have also shown that the thermoelastic stress confinement is essential to generate a strong shock wave. ${ }^{22,27,28,35}$. This confinement should be very weak if we focus a nanosecond pulse in a volume of $\sim 1 \mu \mathrm{m}^{3}$, because the extent of the stress confinement is determined by a condition of $\tau_{\text {heat }}<\tau_{a c}$, where $\tau_{\text {heat }}$ is the heating time of the material, and $\tau_{a c}$ is the characteristic time of acoustic relaxation. ${ }^{28}$ If the time scale of the energy transfer between electron and phonon is as fast as several picoseconds, the heating time of the material is comparable to the pulse duration of the pump laser. On the other hand, $\tau_{a c}$ is an order of acoustic transit time from the heated region. In this study, typically $w_{\text {temp }}=1 \mu \mathrm{m}$ and $v_{a c}=6 \mu \mathrm{m} / \mathrm{ns}$; hence $\tau_{a c}$ is about $160 \mathrm{ps}$. This result means that only a weak pressure wave is generated, if we use a nanosecond-pulsed laser. In order to create a comparable pressure wave by a nanosecond pulse as that by a femotosecond pulse, the total energy of the nanosecond laser light should be much larger, and the focusing of this light may result in a cracking at the irradiated region.

The phase distributions at 2000 and 5000 ps in Fig. 9 indicate that the density in the central region decreased by the thermal expansion and the surrounding region is compressed. This low density due to the localized high temperature should disappear by the thermal diffusion process in a few microseconds. It is important to note here that only a localized high-temperature distribution, which can be achieved by a nanosecond laser pulse, cannot account for the permanent refractive-index increase. Hence, the structural change in the picosecond time range should be important for the cause of the permanent refractive-index increase, although the refractive-index change in the central region is still negative in this time range due to the thermal expansion effect. In other words, the cause of the structural change that leads to the permanent refractive-index increase is already formed on this fast time scale, and the glass structure, which may possess a positive refractive-index change remains and becomes apparent as the permanent change after the thermal energy is diffused out from the irradiated region in the range of a few microseconds.

In this study, we found two phases of the refractive-index increase within a nanosecond (the broken line in Fig. 10); at 20-100 and 500-700 ps, in the laser focal region inside a glass. The first change may be induced by the conformational change at high temperature under high pressure in the confined region as discussed in Secs. IV C and IV D. This change may remain even after the pressure decreases by the acoustic propagation from the irradiated region and also after the temperature decreases by the thermal diffusion process in a later time to produce the permanent refractive-index change.

The transient density increase at $500-700$ ps, which is observed in the result of the thermoelastic calculation [Fig. $5(\mathrm{c})]$, is explained in terms of the recovery from the rapid thermal expansion of the central material. In order to examine the effect of the laser pulse duration on the density change at the center, we calculated the thermoelastic relaxation of the material after the heating of the volume with 1 $\mu \mathrm{m}$ in a radius with various heating times $\left(\tau_{\text {heat }}\right)$. The calculation was conducted by the same method described in Sec. IV B. The temporal evolutions of the density change at the center with various $\tau_{\text {heat }}$ are shown in Fig. 11 . When $\tau_{\text {heat }}$ is as short as 10 ps, the density at the center decreases first 200 ps but recovers from 200 to 500 ps. As $\tau_{\text {heat }}$ becomes longer, the density recovery becomes smaller and disappears with $\tau_{\text {heat }} \geqslant 200 \mathrm{ps}$. It indicates that the heating time have to be as short as 10 ps to maximize the density recovery. Therefore, this density recovery is also a unique phenomena for focusing of the femtosecond-laser pulse.

Chan et al. also suggest that their observation of Raman band change may reflect the shock wave and the shock wave should induce densification of the irradiated glass. ${ }^{7}$ Our observation clearly shows the pressure wave and supports their 


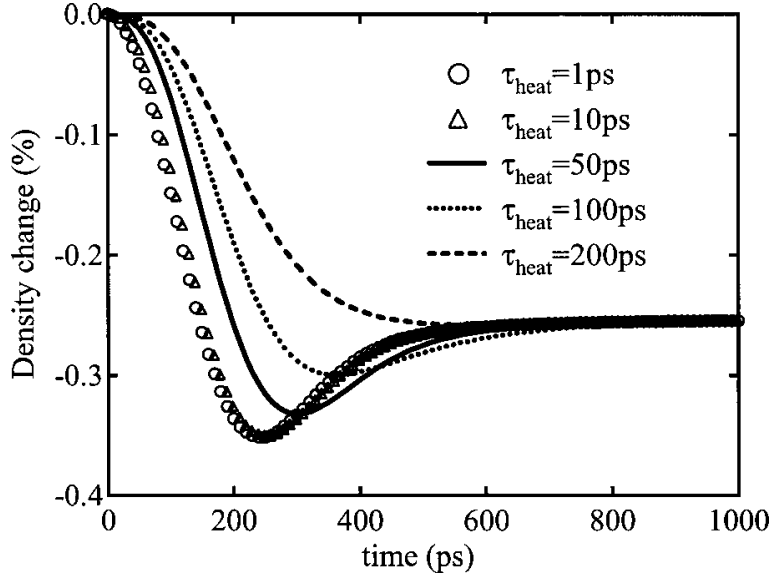

FIG. 11. The calculated density changes at the center of the heated region are shown as a function of the heating times $\left(\tau_{\text {heat }}\right)$. The calculations were conducted using the thermoelastic equation [Eqs. (9)-(12)] with the mechanical constants of the soda-lime glass, $\Delta T=1000 \mathrm{~K}, w_{\text {temp }}=1 \mu \mathrm{m}$, and $\tau_{\text {heat }}=1,10,50,100$, and 200 ps, respectively.

suggestion. The observation in a much longer time range as well as a more elaborate calculation may be necessary for the further investigation of this effect and will be reported in future.

\section{SUMMARY}

The refractive-index change after focusing a femtosecond-pulsed-laser beam in a glass was investigated by the TrL method with a time resolution of subpicoseconds. The TrL signal shows the intensity oscillation with a time period of about 800 ps and the origin of the oscillation was clearly explained in terms of pressure-wave propagation. The spatial and temporal developments of the phase distribution
$\Delta \phi(t, r)$ were calculated from the TrL image using the phaseretrieval method. We found (i) the refractive-index distribution changes immediately after the photoexcitation, (ii) the refractive index increase in 20-100 ps, (iii) the generation and propagation of the pressure wave, (iv) the increase of the density at the center of the laser-irradiated region accompanied by the acoustic wave propagation (500-700 ps), and (v) quasistatic temperature distribution in the laser-irradiated region in the time window of this experiment $(\sim 5000 \mathrm{ps})$. The refractive-index increase at 20-100 ps may be caused by the structural change of the glass at high temperature under high pressure. This fast change in the refractive index and the pressure-wave generation and transient density increase should be unique events induced by the femtosecond laser. This fact indicates that the pressure wave could be an important factor for creating the structural change. Although we do not observe the refractive-index change in nanosecondmicrosecond time range, the cause of the permanent refractive-index change should be already created in the picosecond range of this study. After the thermal diffusion from the irradiated region, the result of the structural change becomes apparent as the permanent refractive-index change which we observed at $10 \mathrm{~s}$ after the irradiation. The observation in this study will open a way to elucidate the mechanism of femtosecond-laser-induced refractive-index increase inside a glass.

\section{ACKNOWLEDGMENTS}

The authors thank Professor K. Hirao, Dr. K. Fujita, and Mr. Y. Shimotsuma of the Kyoto University, Dr. K. Miura of the Central Glass Corporation, Dr. J. Qiu of JST, and Professor M. Obara of the Keio University for fruitful discussion. This work is supported by the Grant-in-Aid (No. 13853002) and the Grant-in-Aid for JSPS from the Ministry of Education, Science, Sports and Culture in Japan.
${ }^{1}$ K. M. Davis, K. Miura, N. Sugimoto, and K. Hirao, Opt. Lett. 21, 1729 (1996).

${ }^{2}$ E. N. Glezer, M. Milosavljevic, L. Huang, R. J. Finlay, T.-H. Her, J. P. Callan, and E. Mazur, Opt. Lett. 21, 2023 (1996).

${ }^{3}$ K. Minoshima, A. M. Kowalevicz, I. Hartl, E. P. Ippen, and J. G. Fujimoto, Opt. Lett. 26, 1516 (2001).

${ }^{4}$ M. Kamata, and M. Obara., Appl. Phys. A: Mater. Sci. Process. 78, 85 (2004).

${ }^{5}$ M. Will, S. Nolte, B. N. Chichkov, and A. Tunnermann, Appl. Opt. 41, 4360 (2002).

${ }^{6}$ A. M. Streltsov and N. F. Borrelli, J. Opt. Soc. Am. B 19, 2496 (2002).

${ }^{7}$ J. W. Chan, T. R. Huster, S. H. Risbud, and D. M. Krol, Appl. Phys. A: Mater. Sci. Process. 76, 367 (2003).

${ }^{8}$ C. B. Schaffer, J. F. Garcia, and E. Mazur, Appl. Phys. A: Mater. Sci. Process. 76, 351 (2003).

${ }^{9}$ C. B. Schaffer, A. O. Jamison, and E. Mazur, Appl. Phys. Lett. 84, 1441 (2004).

${ }^{10}$ H.-B. Sun, V. Mizeikis, Y. Xu, S. Juodkazis, J.-Y Ye, S. Matsuo, and H. Misawa, Appl. Phys. Lett. 79, 1 (2001).

${ }^{11}$ W. Watanabe, T. Toma, K. Yamada, J. Nishii, K. Hayashi, and K. Itoh, Opt. Lett. 25, 1669 (2000).

${ }^{12}$ M. Watanabe, S. Juodkazis, H-B. Son, S. Matsuo, and H. Misawa, Phys. Rev. B 60, 9959 (1999).

${ }^{13}$ L. Sudrie, M. Franco, B. Prade, and A. Mysyrowicz, Opt. Commun., 191, 333 (2001).

${ }^{14}$ C. B. Schaffer, A. Brodeur, and E. Mazur, Meas. Sci. Technol. 12, 1784 (2001).

${ }^{15}$ P. Martin, S. Guizard, Ph. Daguzan, G. Petite, P. D'Oliveira, P. Meynadier, and M. Perdrix, Phys. Rev. B 55, 5799 (1997).

${ }^{16}$ S. Sen and J. E. Dickinson, Phys. Rev. B 68, 214204 (2003).

${ }^{17}$ M. Sakakura and M. Terazima., Opt. Lett. 29, 1548 (2004).

${ }^{18}$ M. Terazima, T. Hara, and N. Hirota, J. Phys. Chem. 93, 13668 (1993).

${ }^{19}$ M. Terazima, Opt. Lett. 20, 25 (1995).

${ }^{20}$ C. Hu. and J. R. Whinnery, Appl. Opt. 12, 72 (1973).

${ }^{21}$ J. F. Power, Appl. Opt. 29, 52 (1990).

${ }^{22}$ B. C. Stuart, M. D. Feit, A. M. Rubenchik, B. W. Shore, and M. 
D. Perry, Phys. Rev. Lett. 74, 2248 (1995).

${ }^{23}$ K. Murata, KOGAKU (Science Co., Tokyo, 1980).

${ }^{24}$ K. Iizuka, Engineering Optics (Springer-Verlag, Berlin, 1985).

${ }^{25}$ J. R. Fienup, Appl. Opt. 21, 2758 (1982).

${ }^{26}$ W. H. Press, B. P. Flannery, A. A. Teukolsky and W. T. Vetterling, Numerical Recipes in C: The art of Scientific Computing (Cambridge University Press, Cambridge, 1988).

${ }^{27}$ I. Itzkan, D. Albagli, M. Dark, L. Perelman, C. von Rosenberg, and M. S. Feld, Proc. Natl. Acad. Sci. U.S.A. 92, 1960 (1995).

${ }^{28}$ G. Paltauf and P. E. Dyer, Chem. Rev. (Washington, D.C.) 103, 487 (2003).

${ }^{29}$ L. D. Landau, and E. M. Lifshitz, Theory of Elasticity (Pergamon,
Oxford, 1986).

${ }^{30}$ O. C. Zienkiewicz, K. Morgan, Finite Elements and Approximation (Wiley, New York, 1983).

${ }^{31}$ Z. Rosenberg, N. K. Bourne, and J. C. F. Millett, J. Appl. Phys. 79, 3971 (1996).

${ }^{32}$ J. R. Rustad, D. A. Yuen and F. J. Spera, Phys. Rev. B 44, 2108 (1991).

${ }^{33}$ S. Spinner, J. Am. Ceram. Soc. 39, 113 (1956).

${ }^{34}$ L. P. Davila, M.-J. Caturla, A. Kubota, B. Sadigh, T. D. Rubia, J. F. Shackelford, S. H. Risbud, and S. H. Garofalini, Phys. Rev. Lett. 91, 205501 (2003).

${ }^{35}$ D. S. Ivanov and L. V. Zhigilei, Phys. Rev. B 68, 064114 (2003). 